\title{
A Recurrence for Counting Graphical Partitions
}

\author{
Tiffany M. Barnes * \\ Carla D. Savage ${ }^{\dagger}$ \\ Department of Computer Science \\ North Carolina State University \\ Raleigh, North Carolina 27695-8206
}

Submitted: February 2, 1995; Accepted May 18, 1995.

\begin{abstract}
In this paper, we give a recurrence to enumerate the set $G(n)$ of partitions of a positive even integer $n$ which are the degree sequences of simple graphs. The recurrence gives rise to an algorithm to compute the number of elements of $G(n)$ in time $O\left(n^{4}\right)$ using space $O\left(n^{3}\right)$. This appears to be the first method for computing $|G(n)|$ in time bounded by a polynomial in $n$, and it has enabled us to tabulate $|G(n)|$ for even $n \leq 220$.
\end{abstract}

\section{Introduction}

A partition of a positive integer $n$ is a sequence of positive integers $\left(\pi_{1}, \pi_{2}, \ldots, \pi_{l}\right)$ satisfying $\pi_{1} \geq \pi_{2} \geq \ldots \geq \pi_{l}$ and $\pi_{1}+\pi_{2}+\ldots+\pi_{l}=n$. Let $P(n)$ denote the set of all partitions of n. $P(0)$ contains only the empty partition, $\lambda$. A partition $\pi \in P(n)$ is graphical if it is the degree sequence of some simple undirected graph. For example, $(5,4,4,3,3,1)$ is the degree sequence of the graph in Figure 1(a), but $(5,4,4,2,2,1)$ is not graphical. Clearly, graphical partitions exist only when $n$ is even, since the sum of the degrees of the vertices of a graph is equal to twice the number of edges. Let $G(n)$ denote the set of graphical partitions of $n$. For convenience, we will call the empty partition graphical, so that $|G(0)|=1$.

Several necessary and sufficient conditions to determine whether an integer sequence is graphical are surveyed in $[\mathrm{SH}]$. Perhaps the best known is the following condition due to Erdös and Gallai [EG]:

\footnotetext{
${ }^{*}$ Research supported by the National Science Foundation through the CRA Distributed Mentor Project, 1994

${ }^{\dagger}$ Research supported in part by National Science Foundation Grant No. CCR8906500 and DIMACS
} 


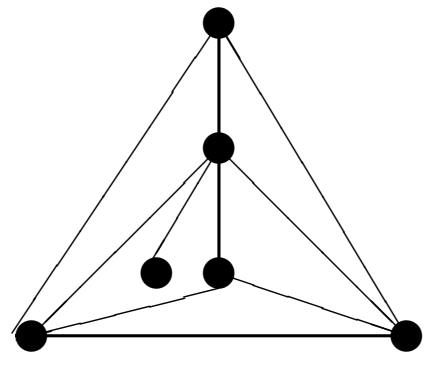

(a)

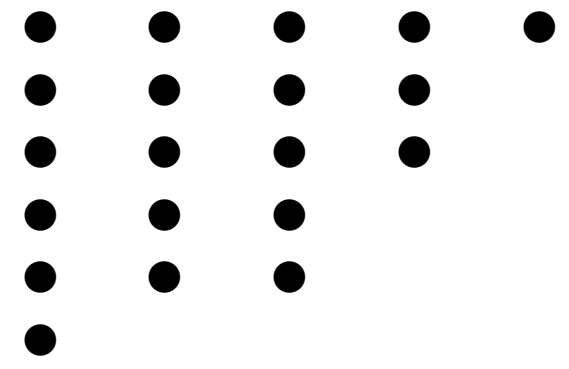

(b)

Figure 1: (a) A graph with degree sequence $\pi=(5,4,4,3,3,1)$ and (b) the Ferrars graph of $\pi$.

[Erdös - Gallai] A positive integer sequence $\left(\pi_{1}, \pi_{2}, \ldots, \pi_{l}\right)$, with $\pi_{1} \geq \pi_{2} \geq \ldots \geq \pi_{l}$, is graphical if and only if $\pi_{1}+\pi_{2}+\ldots+\pi_{l}$ is even and for $1 \leq j \leq l$,

$$
\sum_{i=1}^{j} \pi_{i} \leq j(j-1)+\sum_{i=j+1}^{l} \min \left\{j, \pi_{i}\right\} .
$$

In Section 2, we use a lesser-known condition to devise a recurrence to enumerate $G(n)$. As shown in Section 3, it can be used to count $G(n)$ in time $O\left(n^{4}\right)$ using space $O\left(n^{3}\right)$.

Our work was motivated by the following question, originally posed by Herbert Wilf, which remains open:

[Question] What fraction of the elements of $P(n)$ are graphic? In particular, does the ratio $|G(n)| /|P(n)|$ approach 0 as $n$ approaches infinity?

To even plot the ratio $|G(n)| /|P(n)|$, it is necessary to compute $|G(n)|$, which, in our initial attempts, became a computational burden well before $n=100$. Using an earlier version of the recurrence, we were able to compute $|G(n)|$ up to $n=220$. These results are tabulated in Section 4. Where sufficient memory is available, computing $|G(n)|$ up to $n=1000$ should be feasible.

For a related counting problem, we note that Stanley [St] has obtained a generating function for $f(n)$, the number of sequences $\left(x_{1}, x_{2}, \ldots, x_{n}\right)$ which are degree sequences of simple graphs with vertex set $\left\{v_{1}, v_{2}, \ldots, v_{n}\right\}$. Here, $x_{i}$ is the degree of vertex $v_{i}$ and the degree sequence is not necessarily nonincreasing. 
THE ELECTRONIC JOURNAL OF COMBINATORICS 2 (1995), \#R11

\section{The Recurrence}

For a partition $\pi=\left(\pi_{1}, \ldots, \pi_{l}\right)$, the associated Ferrars graph is an array of $l$ rows of dots, where row $i$ has $\pi_{i}$ dots and rows are left justified (Figure 1(b).) Let $\pi^{\prime}$ denote the conjugate partition $\pi^{\prime}=\left(\pi_{1}^{\prime}, \ldots, \pi_{m}^{\prime}\right)$ where $m=\pi_{1}$ and $\pi_{i}^{\prime}$ is the number of dots in the $i$-th column of the Ferrars graph of $\pi$. The Durfee square of $\pi$ is the largest square subarray of dots in the Ferrars graph of $\pi$. Let $d(\pi)$ denote the size (number of rows) of the Durfee square of $\pi$. The sequence

$$
\left(\pi_{1}-\pi_{1}^{\prime}, \quad \pi_{2}-\pi_{2}^{\prime}, \ldots, \pi_{d(\pi)}-\pi_{d(\pi)}^{\prime}\right)
$$

is the sequence of successive ranks of $\pi$ [At]. It will be convenient to work with the negatives of the ranks, so, for $1 \leq i \leq d(\pi)$, let $r_{i}(\pi)=\pi_{i}^{\prime}-\pi_{i}$. We call $\left(r_{1}(\pi), \ldots r_{d(\pi)}(\pi)\right)$ the sequence of successive antiranks of $\pi$.

The necessary and sufficient condition below, attributed to Nash-Williams, is proved in [RA2] and [SH].

[Nash-Williams] A partition $\pi$ of an even integer is graphical if and only if for $1 \leq j \leq$ $d(\pi)$,

$$
\sum_{i=1}^{j} r_{i}(\pi) \geq j .
$$

(This condition is called the Hässelbarth Criterion by the authors of [SH] since they first saw it in [Has], where it appeared without proof.) It can be shown that for $1 \leq j \leq d(\pi)$, the $j$-th Nash-Williams condition is equivalent to the $j$-th Erdös-Gallai condition. Furthermore, if conditions $1,2, \ldots, d(\pi)$ of Erdös-Gallai are satisfied, then so are the remaining Erdös-Gallai conditions $[\mathrm{RA} 2]$.

Let $P(n, k, l)$ be the set of partitions of $n$ into at most $l$ parts with largest part at most $k$ and define $G(n, k, l)$ to be the set of graphical partitions in $P(n, k, l)$. Let $\pi \in P(n, k, l)$ and let $\alpha$ be obtained from $\pi$ by deleting the first row and column of the Ferrars graph of $\pi$. Then $d(\alpha)=d(\pi)-1$. Let $s=r_{1}(\pi)=\pi_{1}^{\prime}-\pi_{1}$. By the Nash-Williams condition, $\pi$ is graphical if and only if $s>0$ and for $1 \leq j \leq d(\alpha)$, the antiranks of $\alpha$ satisfy an $s$-variant of the Nash-Williams conditions:

$$
s+\sum_{i=1}^{j} r_{i}(\alpha) \geq j .
$$


With this in mind, define $P(n, k, l, s)$ for $s \geq 0$ by

$$
P(n, k, l, s)=\left\{\pi \in P(n, k, l) \mid s+\sum_{i=1}^{j} r_{i}(\pi) \geq j \text { for } 1 \leq j \leq d(\pi)\right\} .
$$

Let $P(n, k, l, s)=\emptyset$ if $s<0$ and note that for $s \geq 0, P(n, k, l, s)=\{\lambda\}$ if $P(n, k, l)=\{\lambda\}$.

Lemma 1 below is a restatement of the Nash-Williams condition and Lemma 2 follows since $G(n)=G(n, n, n)$.

Lemma 1 For even $n \geq 0, G(n, k, l)=P(n, k, l, 0)$.

Lemma 2 For even $n \geq 0, G(n)=P(n, n, n, 0)$.

Thus, we can compute $|G(n)|$ by computing $|P(n, k, l, s)|$ for appropriate values of the arguments. To this end, let $P^{\prime}(n, k, l)$ and $P^{\prime}(n, k, l, s)$, be the subsets of $P(n, k, l)$ and $P(n, k, l, s)$, respectively, consisting of those partitions into exactly $l$ parts with largest part of size exactly $k$.

Lemma 3 For $n>0$ and $1 \leq k, l, s \leq n$,

$|P(n, k, l, s)|-\left|P^{\prime}(n, k, l, s)\right|=|P(n, k-1, l, s)|+|P(n, k, l-1, s)|-|P(n, k-1, l-1, s)|$.

Proof. From the definitions of $P$ and $P^{\prime}$ we have

$$
P(n, k, l, s) \backslash P^{\prime}(n, k, l, s)=P(n, k-1, l, s) \cup P(n, k, l-1, s) .
$$

The set on the left-hand side of this equality has size

$$
|P(n, k, l, s)|-\left|P^{\prime}(n, k, l, s)\right|
$$

and by inclusion-exclusion, the set on the right-hand side of the equality has size

$$
|P(n, k-1, l, s)|+|P(n, k, l-1, s)|-|P(n, k-1, l, s) \cap P(n, k, l-1, s)| .
$$

The result follows since the intersection in the last term is $P(n, k-1, l-1, s)$.

Lemma 4 Assume $n>0,1 \leq k, l, \leq n$, and $s \geq 0$. Then

$$
\left|P^{\prime}(n, k, l, s)\right|=|P(n-k-l+1, \quad k-1, \quad l-1, \quad s+l-k-1)| .
$$


THE ELECTRONIC JOURNAL OF COMBINATORICS 2 (1995), \#R11

Proof. Define a function $f$ on $P^{\prime}(n, k, l)$ by $f(\pi)=\alpha$, where $\alpha$ is obtained from $\pi$ by deleting the first row and column in the Ferrars graph of $\pi$. Given the assumptions of the theorem, if $P^{\prime}(n, k, l)=\emptyset$ then either $(1) n<k+l-1$, in which case $n-k-l+1<0$ or (2) $n>k l$, which implies $n-k-l+1>k l-k-l+1=(k-1)(l-1)$. In either of these cases, $P(n-k-l+1, k-1, l-1)=\emptyset$. If $P^{\prime}(n, k, l)$ contains only the partition $(k, 1, \ldots, 1)$, then $f((k, 1, \ldots, 1))=\lambda, n-k-l+1=0$, and $P(n-k-l+1, k-1, l-1)=\{\lambda\}$. Otherwise, $d(\alpha)=d(\pi)-1$ and $\alpha=\left(\alpha_{1}, \ldots \alpha_{m}\right)$ where $m=\pi_{2}^{\prime}-1, \alpha_{i}=\pi_{i+1}-1$ for $1 \leq i \leq m$ and $\alpha_{i}^{\prime}=\pi_{i+1}^{\prime}-1$ for $1 \leq i \leq d(\pi)-1$. Clearly, $f$ is a bijection between $P^{\prime}(n, k, l)$ and $P(n-k-l+1, k-1, l-1)$ Furthermore, for $1 \leq j \leq d(\pi)$,

$$
\begin{aligned}
s+\sum_{i=1}^{j}\left(\pi_{i}^{\prime}-\pi_{i}\right) & =(s+l-k)+\sum_{i=2}^{j}\left(\pi_{i}^{\prime}-\pi_{i}\right) \\
& =(s+l-k)+\sum_{i=2}^{j}\left(\left(\pi_{i}^{\prime}-1\right)-\left(\pi_{i}-1\right)\right) \\
& =(s+l-k)+\sum_{i=1}^{j-1}\left(\alpha_{i}^{\prime}-\alpha_{i}\right) .
\end{aligned}
$$

Thus

$$
\begin{gathered}
s+\sum_{i=1}^{j} r_{i}(\pi) \geq j \Longleftrightarrow \\
(s+l-k-1)+\sum_{i=1}^{j-1} r_{i}(\alpha) \geq j-1 .
\end{gathered}
$$

This establishes that $\pi \in P^{\prime}(n, k, l, s) \Longleftrightarrow \alpha \in P(n-k-l+1, k-1, l-1, s+l-k-1)$.

Lemma $5 P(n, k, l)=P(n, k, l, n)=P(n, k, l, s)$ for $s \geq n$.

Proof. Note that for any $\pi \in P(n, k, l)$ and $1 \leq j \leq \pi(d)$,

$$
\sum_{i=1}^{j}\left(\pi_{i}^{\prime}-\pi_{i}-1\right) \geq \sum_{i=1}^{j}-\pi_{i} \geq-n .
$$

Thus, $\pi \in P(n, k, l, n)$, which means $P(n, k, l) \subseteq P(n, k, l, n)$. By definition, for $t^{\prime} \geq t \geq 0$, $P(n, k, l, t) \subseteq P\left(n, k, l, t^{\prime}\right)$, thus for any $s \geq n, P(n, k, l, n) \subseteq P(n, k, l, s)$. The result follows since $P(n, k, l, s) \subseteq P(n, k, l)$.

The resulting recurrence for counting $|P(n, k, l, s)|$ is given in the following. 
Theorem $1|P(n, k, l, s)|$ is defined by:

$$
\begin{aligned}
& |P(n, k, l, s)|= \\
& \text { if }((n<0) \text { or }(k<0) \text { or }(l<0) \text { or }(s<0)) \quad \text { then : } 0 \\
& \text { else if } n=0 \quad \text { then: } 1 \\
& \text { else if }(k=0) \text { or }(l=0) \quad \text { then: } 0 \\
& \text { else if }(k>n) \quad \text { then: }|P(n, n, l, s)| \\
& \text { else if }(l>n) \quad \text { then: }|P(n, k, n, s)| \\
& \text { else if }(s>n) \quad \text { then: }|P(n, k, l, n)| \\
& \text { else: }|P(n, k-1, l, s)|+|P(n, k, l-1, s)|-|P(n, k-1, l-1, s)| \\
& +|P(n-k-l+1, k-1, l-1, s+l-k-1)|
\end{aligned}
$$

Proof. $P(n, k, l, s)$ was defined to be empty when $s<0$. For the remaining conditions in (1) through (5) the value of $|P(n, k, l, s)|$ is clear. Condition (6) follows from Lemma 5 . For the general case (7), equate $\left|P^{\prime}(n, k, l, s)\right|$ in Lemmas 3 and 4 and then solve for $|P(n, k, l, s)|$.

\section{The Algorithm}

The recurrence of Theorem 1 for computing $|P(n, k, l, s)|$ has a straightforward implementation as a dynamic programming algorithm which fills a 4-dimensional table of entries $T[a, b, c, d]=|P(a, b, c, d)|$ where $0 \leq a \leq n, 0 \leq b \leq k, 0 \leq c \leq l$, and $0 \leq d \leq n$. The table is filled in any order which guarantees that when the time comes to fill entry $T\left[n^{\prime}, k^{\prime}, l^{\prime}, s^{\prime}\right]$, the required entries $T\left[n^{\prime}, k^{\prime}-1, l^{\prime}, s^{\prime}\right], T\left[n^{\prime}, k^{\prime}, l^{\prime}-1, s^{\prime}\right], T\left[n^{\prime}, k^{\prime}-1, l^{\prime}-1, s^{\prime}\right]$, and $T\left[n^{\prime}-k^{\prime}-l^{\prime}+1, k^{\prime}-1, l^{\prime}-1, s^{\prime}+l^{\prime}-k^{\prime}-1\right]$ have already been filled and can be read from the table. The table uses space $O\left(n^{2} k l\right)$ and only constant time is required to fill in each entry. In particular, computing $|G(n)|=|P(n, n, n, 0)|$ takes time and space $O\left(n^{4}\right)$.

The space can be asymptotically improved as follows. For $0 \leq c \leq l$, let $T_{c}$ be the 3 -dimensional table of entries $T_{c}[a, b, d]=|P(a, b, c, d)|$ for $0 \leq a \leq n, 0 \leq b \leq k$, and $0 \leq d \leq n$. Then $|P(n, k, l, s)|$ can be computed by computing successively the tables $T_{0}, T_{1}, \ldots T_{l}$. Note from the recurrence of Theorem 1 that computing entries in table $T_{c}$ requires access only to values in table $T_{c}$ or table $T_{c-1}$. Thus, in computing $|P(n, k, l, s)|$, no more than two 3-dimensional tables need to be stored at any given time, reducing the 
THE ELECTRONIC JOURNAL OF COMBINATORICS 2 (1995), \#R11

space required to $O\left(n^{2} k\right)$. Thus, computing $|G(n)|$ can be done in $O\left(n^{4}\right)$ time with $O\left(n^{3}\right)$ space.

\section{Concluding Remarks}

Even with this polynomial time algorithm, computing $|G(n)|$ for $n>200$ quickly becomes impractical because of the huge space requirements. An additional burden on space is that $|G(n)|$ gets large quickly so that some method must be used to manipulate and allocate enough storage for these large numbers. The following strategy was suggested by the referee: Select small primes $p_{1}<p_{2}<\ldots<p_{s}$ so that $p_{1} p_{2} \ldots p_{s}>G(n)$. For $i=1, \ldots, s$, use the recurrence of Theorem 1 to compute $G_{i}(n)=G(n) \bmod \left(p_{i}\right)$. Then by the Chinese Remainder Theorem, $G(n)$ can be recovered from $G_{1}(n), \ldots, G_{s}(n)$. If, for example, the primes can be represented with 8 bits, time $O\left(n^{4}\right)$ will be spent computing each of $s$ tables, but the 3-dimensional tables now need store only 8-bit integers.

For those interested in the values $|G(n)|$, or in the ratio $|G(n)| /|P(n)|$ from the open question of Section 1, we include Tables 1 and 2. To the best of our knowledge, the values had previously been computed only through $n=40$, as noted in [ER] in an acknowledgement to Ron Read. From the data, it seems reasonable to make the conjecture that for even $n \geq 18$, $|G(n)| /|P(n)|$ is monotone decreasing, but we are not aware of any proof of this. The best results known at this time are that

$$
\varlimsup_{n \rightarrow \infty} \frac{\sqrt{n}|G(n)|}{|P(n)|} \geq \frac{\pi}{\sqrt{6}}
$$

(so the ratio cannot go to 0 faster than $1 / \sqrt{n}[\mathrm{ER}]$ ) and that [RA1]

$$
\varlimsup_{n \rightarrow \infty} \frac{|G(n)|}{|P(n)|} \leq .25
$$

Acknowledgements: Thanks to Steve Skiena for bringing this problem to our attention, to Frank Ruskey for pointing out references [SH] and [St], and to Bruce Richmond and Cecil Rousseau for sharing their work with us. A special thanks to Herbert Wilf for his help in simplifying a earlier version of our recurrence which involved a double summation. This led to asymptotic improvements in both the time and space required. We are also grateful to the referees for their insightful comments and helpful suggestions. 


\begin{tabular}{|l||l|l|l|}
\hline$n$ & $|G(n)|$ & $|P(n)|$ & $|G(n)| /|P(n)|$ \\
\hline \hline 2 & 1 & 2 & 0.500000 \\
\hline 4 & 2 & 5 & 0.400000 \\
\hline 6 & 5 & 11 & 0.454545 \\
\hline 8 & 9 & 22 & 0.409091 \\
\hline 10 & 17 & 42 & 0.404762 \\
\hline 12 & 31 & 77 & 0.402597 \\
\hline 14 & 54 & 135 & 0.400000 \\
\hline 16 & 90 & 231 & 0.389610 \\
\hline 18 & 151 & 385 & 0.392208 \\
\hline 20 & 244 & 627 & 0.389155 \\
\hline 22 & 387 & 1002 & 0.386228 \\
\hline 24 & 607 & 1575 & 0.385397 \\
\hline 26 & 933 & 2436 & 0.383005 \\
\hline 28 & 1420 & 3718 & 0.381926 \\
\hline 30 & 2136 & 5604 & 0.381156 \\
\hline 32 & 3173 & 8349 & 0.380046 \\
\hline 34 & 4657 & 12310 & 0.378310 \\
\hline 36 & 6799 & 17977 & 0.378205 \\
\hline 38 & 9803 & 26015 & 0.376821 \\
\hline 40 & 14048 & 37338 & 0.376239 \\
\hline 42 & 19956 & 53174 & 0.375296 \\
\hline 44 & 28179 & 75175 & 0.374845 \\
\hline 46 & 39467 & 105558 & 0.373889 \\
\hline 48 & 54996 & 147273 & 0.373429 \\
\hline 50 & 76104 & 204226 & 0.372646 \\
\hline 52 & 104802 & 281589 & 0.372181 \\
\hline 54 & 143481 & 386155 & 0.371563 \\
\hline 56 & 195485 & 526823 & 0.371064 \\
\hline 58 & 264941 & 715220 & 0.370433 \\
\hline 60 & 357635 & 966467 & 0.370044 \\
\hline 62 & 480408 & 1300156 & 0.369500 \\
\hline 64 & 642723 & 1741630 & 0.369035 \\
\hline 66 & 856398 & 2323520 & 0.368578 \\
\hline 68 & 1136715 & 3087735 & 0.368139 \\
\hline 70 & 1503172 & 4087968 & 0.367706 \\
\hline 72 & 1980785 & 5392783 & 0.367303 \\
\hline 74 & 2601057 & 7089500 & 0.366889 \\
\hline 76 & 3404301 & 9289091 & 0.366484 \\
\hline 78 & 4441779 & 12132164 & 0.366116 \\
\hline 80 & 5777292 & 15796476 & 0.365733 \\
\hline 82 & 7492373 & 20506255 & 0.365370 \\
\hline 84 & 9688780 & 26543660 & 0.365013 \\
\hline 86 & 12494653 & 34262962 & 0.364669 \\
\hline 88 & 16069159 & 44108109 & 0.364313 \\
\hline 90 & 20614755 & 56634173 & 0.363999 \\
\hline 92 & 26377657 & 72533807 & 0.363660 \\
\hline 94 & 33671320 & 92669720 & 0.363348 \\
\hline 96 & 42878858 & 118114304 & 0.363028 \\
\hline 98 & 54481054 & 150198136 & 0.362728 \\
\hline 100 & 69065657 & 190569292 & 0.362418 \\
\hline 102 & 87370195 & 241265379 & 0.362133 \\
\hline 104 & 110287904 & 304801365 & 0.361835 \\
\hline 106 & 138937246 & 384276336 & 0.361556 \\
\hline 108 & 174675809 & 483502844 & 0.361272 \\
\hline 110 & 219186741 & 607163746 & 0.361001 \\
\hline & & & \\
\hline
\end{tabular}

Table 1: Sizes of $G(n)$ and $P(n)$ and their ratio for $2 \leq n \leq 110$. 


\begin{tabular}{|c|c|c|c|}
\hline$n$ & $|G(n)|$ & $|P(n)|$ & $|G(n)| / \mid P(n)$ \\
\hline$\overline{112}$ & 274512656 & $\overline{761002156}$ & 0.360725 \\
\hline 114 & 343181668 & 952050665 & 0.360466 \\
\hline 116 & 428244215 & 1188908248 & 0.360200 \\
\hline 118 & 533464959 & 1482074143 & 0.359945 \\
\hline 120 & 663394137 & 1844349560 & 0.359690 \\
\hline 122 & 823598382 & 2291320912 & 0.359443 \\
\hline 124 & 1020807584 & 2841940500 & 0.359194 \\
\hline 126 & 1263243192 & 3519222692 & 0.358955 \\
\hline 128 & 1560795436 & 4351078600 & 0.358715 \\
\hline 130 & 1925513465 & 5371315400 & 0.358481 \\
\hline 132 & 2371901882 & 6620830889 & 0.358248 \\
\hline 134 & 2917523822 & 8149040695 & 0.358021 \\
\hline 136 & 3583515700 & 10015581680 & 0.357794 \\
\hline 138 & 4395408234 & 12292341831 & 0.357573 \\
\hline 140 & 5383833857 & 15065878135 & 0.357353 \\
\hline 142 & 6585699894 & 18440293320 & 0.357136 \\
\hline 144 & 8045274746 & 22540654445 & 0.356923 \\
\hline 146 & 9815656018 & 27517052599 & 0.356711 \\
\hline 148 & 11960467332 & 33549419497 & 0.356503 \\
\hline 150 & 14555902348 & 40853235313 & 0.356297 \\
\hline 152 & 17692990183 & 49686288421 & 0.356094 \\
\hline 154 & 21480510518 & 60356673280 & 0.355893 \\
\hline 156 & 26048320019 & 73232243759 & 0.355695 \\
\hline 158 & 31551087790 & 88751778802 & 0.355498 \\
\hline 160 & 38173235010 & 107438159466 & 0.355304 \\
\hline 162 & 46134037871 & 129913904637 & 0.355112 \\
\hline 164 & 55694314567 & 156919475295 & 0.354923 \\
\hline 166 & 67163674478 & 189334822579 & 0.354735 \\
\hline 168 & 80909973315 & 228204732751 & 0.354550 \\
\hline 170 & 97368672089 & 274768617130 & 0.354366 \\
\hline 172 & 117056456152 & 330495499613 & 0.354185 \\
\hline 174 & 140584220188 & 397125074750 & 0.354005 \\
\hline 176 & 168675124141 & 476715857290 & 0.353827 \\
\hline 178 & 202182888436 & 571701605655 & 0.353651 \\
\hline 180 & 242116891036 & 684957390936 & 0.353477 \\
\hline 182 & 289666252014 & 819876908323 & 0.353305 \\
\hline 184 & 346234896845 & 980462880430 & 0.353134 \\
\hline 186 & 413474657328 & 1171432692373 & 0.352965 \\
\hline 188 & 493331835384 & 1398341745571 & 0.352700 \\
\hline 190 & 588093594457 & 1667727404093 & 0.352632 \\
\hline 192 & 700451190712 & 1987276856363 & 0.352468 \\
\hline 194 & 833561537987 & 2366022741845 & 0.352305 \\
\hline 196 & 991134281267 & 2814570987591 & 0.352144 \\
\hline 198 & 1177516049387 & 3345365983698 & 0.351984 \\
\hline 200 & 1397805210533 & 3972999029388 & 0.351826 \\
\hline 202 & 1657968320899 & 4714566886083 & 0.351669 \\
\hline 204 & 1964994991232 & 5590088317495 & 0.351514 \\
\hline 206 & 2327052859551 & 6622987708040 & 0.351360 \\
\hline 208 & 2753697110356 & 7840656226137 & 0.351207 \\
\hline 210 & 3256081386335 & 9275102575355 & 0.351056 \\
\hline 212 & 3847232865612 & 10963707205259 & 0.350757 \\
\hline 214 & 4542341563460 & 12950095925895 & 0.350757 \\
\hline 216 & 5359127512113 & 15285151248481 & 0.350610 \\
\hline 218 & 6318223879596 & 18028182516671 & 0.350464 \\
\hline 220 & 7443670977177 & 21248279009367 & 0.350319 \\
\hline
\end{tabular}

Table 2: Sizes of $G(n)$ and $P(n)$ and their ratio for $112 \leq n \leq 220$. 
THE ELECTRONIC JOURNAL OF COMBINATORICS 2 (1995), \#R11

\section{References}

[At] A. O. L. Atkin, "A note on ranks and conjugacy of partitions," Quart. J. Math., Oxford Ser. (2)17 (1966) 335-338.

[EG] P. Erdös and T. Gallai, "Graphs with given degree of vertices," Mat. Lapok 11 (1960) 264-274.

[ER] P. Erdös and L. B. Richmond, "On graphical partitions," Combinatorica 13 (1993) 57-63.

[Has] W. Hässelbarth, "Die Verzweigtheit von Graphen", Match. 16 (1984) 3-17.

[RA1] C. C. Rousseau and F. Ali, "On a conjecture concerning graphical partitions," preprint, April 1994.

[RA2] C. C. Rousseau and F. Ali, "A note on graphical partitions," preprint, April 1994.

[SH] G. Sierksma and H. Hoogeveen, "Seven criteria for integer sequences being graphic," Journal of Graph Theory 15, No. 2 (1991) 223-231.

[St] R. P. Stanley, "A zonotope associated with graphical degree sequences," in $A p$ plied Geometry and Discrete Mathematics: The Victor Klee Festschrift, P. Gritznann and B. Sturmfels, eds., ACM, AMS (1991). 